\title{
Associação entre deficiência de vitamina D, adiposidade e exposição solar em participantes do sistema de hipertensão arterial e diabetes melito
}

\section{Association between vitamin D deficiency, adiposity and solar exposure in participants with arterial hypertension and diabetes mellitus}

\author{
Fabiana Silva Ruiz'; Ana Flávia Oliveira²; Andréa Name Colado Simão ${ }^{3}$; Marcell \\ Alysson Batisti Lozovoy ${ }^{4}$; Daniela Frizon Alfieri ${ }^{5}$; Fabiano Sandrini ${ }^{6}$; Isaias Dichi ${ }^{7}$; \\ Jane Bandeira Dichi ${ }^{8}$
}

\begin{abstract}
Resumo
Atualmente, diversos estudos demonstram uma relação entre deficiência de vitamina $\mathrm{D}$ e diabetes melito tipo 2, obesidade e hipertensão arterial. A principal causa de deficiência de vitamina $\mathrm{D}$ é a falta de exposição solar adequada. O objetivo deste estudo foi avaliar os níveis séricos de vitamina $\mathrm{D}$ e sua associação com ingestão de vitamina $\mathrm{D}$, composição corporal e exposição solar em pacientes participantes do Sistema de Hipertensão e Diabetes da cidade de Cascavel, PR. Participaram 304 adultos e idosos, de ambos os sexos, e foram avaliados os dados demográficos e antropométricos, hábitos de vida, presença de doenças prévias, dietética e a dosagem de vitamina D sérica. Utilizou-se do teste qui quadrado para verificação de associação e aderência e o teste de Kruskal-Wallis para comparação de medianas entre as variáveis estudadas. Verificou-se deficiência sérica de 25 -hidroxivitamina D $[25(\mathrm{OH}) \mathrm{D}](<20 \mathrm{ng} / \mathrm{mL})$ em $52,6 \%$, excesso de peso em $73,4 \%$, aumento na circunferência abdominal em $77,6 \%$ e na percentagem de gordura corporal em $95,6 \%$ dos pacientes. Não houve associação entre a ingestão e os níveis séricos de vitamina D. Houve associação significativa entre circunferência abdominal $(p<0,05)$, hipertensão arterial $(p>0,001)$ e exposição solar inadequada $(p<0,001)$ com os diferentes níveis séricos de vitamina $\mathrm{D}$. Os pacientes com níveis séricos desejáveis de vitamina $\mathrm{D}$ apresentaram menores valores de índice de massa corpórea $(p<0,03)$, de circunferência abdominal $(p<0,01)$ e maior tempo de exposição solar $(p=0,01)$. Este estudo verificou uma alta frequência de hipovitaminose $D$, sendo esta, associada com a diminuição de exposição solar e com adiposidade.
\end{abstract}

Palavras-chave: Vitamina D. Diabetes mellitus. Hipertensão. Obesidade. Ingestão de alimentos.

\footnotetext{
${ }^{1}$ Especialista em Nutrição Humana e Saúde pela Universidade Federal de Lavras, Brasil. Professor titular da Faculdade Assiz Gurgacz. E-mail: fabiana.ruiz@gmail.com

${ }^{2}$ Doutora em Nutrição pela Universidade Federal de São Paulo, Brasil. Professora da Universidade Tecnológica Federal do Paraná, Brasil.anaflavia_nutri@hotmail.com

${ }^{3}$ Doutora em Medicina e Ciências da Saúde. Professor Adjunto do Departamento de Patologia, Análises Clínicas e Toxicologia (PAC) do Centro de Ciências da Saúde da UEL. E-mail: deianame@yahoo.com.br

${ }^{4}$ Doutor em Patologia Experimental pela Universidade Estadual de Londrina. Professor do departamento de Análises Clínicas e Toxicologia - Universidade Estadual de Londrina. E-mail: marcell_lozovoy@hotmail.com

${ }^{5}$ Mestranda do Programa de Pós-gradução em Ciências da Saúde pela Universidade Estadual de Londrina.

${ }^{6}$ Doutor em Saúde da Criança e do Adolescente pela Universidade de São Paulo, Brasil. Instituto de Investigação Científica do Paraná

${ }^{7}$ Doutor em Fisiopatologia em Clínica Médica pela Universidade Estadual Paulista Júlio de Mesquita Filho, Brasil. Professor Associado da Universidade Estadual de Londrina

${ }^{8}$ Doutora em Fisiopatologia em Clínica Médica pela Universidade Estadual Paulista Júlio de Mesquita Filho, Brasil. Professor Associado da Universidade Estadual de Londrina
} 


\begin{abstract}
Currently, several studies have shown a relationship between vitamin D deficiency and type 2 diabetes mellitus, obesity and hypertension. The major cause of vitamin $\mathrm{D}$ deficiency is the lack of adequate sun exposure. The objective of the study was to evaluate serum vitamin D level and to verify its association with vitamin D ingestion, body composition and solar exposure in patients participating in the Hypertension and Diabetes System in Cascavel, PR. A total of 304 adult and elder patients from both genders participated in the study. Demographic and anthropometric data, lifestyle, presence of previous diseases, dietary and serum levels of vitamin D were evaluated. We used the chi square test for association verification and compliance and the Kruskal-Wallis test to compare medians between variables. It was verified serum 25 -hydroxyvitamin D $(25(\mathrm{OH}) \mathrm{D})$ deficiency $(<20 \mathrm{ng} / \mathrm{mL})$ in $52.6 \%$, overweight and obesity in $73.4 \%$, increase in abdominal circumference in $77.6 \%$ and in body fat percentage in $95.6 \%$ of the patients. There was no association between ingestion and serum vitamin D levels. Significant association was found between abdominal circumference $(\mathrm{p}<0.05)$, hypertension $(\mathrm{p}=0.0006)$ and inadequate solar exposure $(\mathrm{p}<0.001)$ with serum vitamin $\mathrm{D}$ different levels. There was a high frequency of hypovitaminosis D and association with lower solar exposure and adiposity.
\end{abstract}

Key words: Vitamin D. Diabetes mellitus. Hypertension. Obesity. Eating.

\section{Introdução}

A deficiência de vitamina $\mathrm{D}$ tem sido considerada um problema de saúde pública mundial, atingindo um bilhão de indivíduos (KIMBALL; FULEIHAN; VIETH, 2008). No Brasil, há elevada deficiência desta vitamina nos diversos estudos regionais realizados (CANTOCOSTA; KUNII; HAUACHE, 2006; LIPS et al., 2006; SARAIVA et al., 2007; SILVA et al., 2008). Embora a deficiência de vitamina D esteja relacionada classicamente com o raquitismo e a osteomalácia, atualmente tem-se estudado sua associação com o desenvolvimento de diversas doenças crônicas não transmissíveis (DCNT), entre elas, diabetes melito (DM), obesidade e hipertensão arterial (HA) (PETERLIK; CROSS, 2005; PRENTICE; GOLDBERG; SCHOENMAKERS, 2008).

Sabe-se que DM e HA, doenças causadas por fatores genéticos e ambientais, são responsáveis pelos principais riscos para o desenvolvimento de doenças cardiovasculares (DCV) e se não tratadas adequadamente podem acarretar complicações graves como infarto agudo do miocárdio, insuficiência cardíaca, acidente vascular encefálico, insuficiência renal crônica, amputações de membros inferiores, cegueiras definitivas, abortos e mortes perinatais (BRASIL, 2008). Atualmente, a importância do DM e da HA como problema de saúde pública mundial é bem reconhecida, sobrecarregando a demanda dos sistemas de saúde pública e da previdência social devido, sobretudo à morbimortalidade e elevada invalidez precoce (SOCIEDADE BRASILEIRA DE CARDIOLOGIA, 2010).

Os pacientes com DM e HA no Brasil contam com o apoio do Plano de Reorganização de Atenção à Hipertensão Arterial e Diabetes Melito, chamado de Sistema HiperDia, cujo objetivo principal é oferecer suporte clínico nutricional e medicamentoso a estes pacientes em todo território Nacional (BRASIL, 2008).

Assim, considerando que os diabéticos e hipertensos fazem parte de um grupo de risco de doença cardiovascular e que a detecção e tratamento de deficiência de vitamina $\mathrm{D}$ poderiam contribuir na redução de complicações, este estudo teve como objetivo avaliar os níveis séricos de vitamina $\mathrm{D}$ e verificar associação com a ingestão de vitamina $\mathrm{D}$, o estado nutricional e a exposição solar de pacientes participantes do Sistema de HiperDia da cidade de Cascavel, PR. 


\section{Métodos}

\section{Amostra}

A partir de 4074 indivíduos inscritos no Sistema HiperDia em 23 Unidades Básicas de Saúde (UBS) na cidade de Cascavel, Paraná, foram selecionados 374 indivíduos, de ambos os sexos, com idade entre 33 a 90 anos, com HA e Diabetes Melito tipo 2 (DM2), sendo a amostra calculada com erro amostral de $5 \%$ e intervalo de confiança de 95\%. Com base nesses dados, foram escolhidos por sorteio probabilístico os indivíduos que compunham a amostra de cada UBS, utilizandose tabela de números aleatórios do Programa EXCEL $®$. Nessa etapa, todos os indivíduos sorteados que frequentavam regularmente as reuniões do HiperDia foram selecionados (conglomerados). Durante o estudo, houve 70 perdas e, dessa forma, o total avaliado foi de 304 $(82,1 \%)$ indivíduos (Figura 1).

Todos os participantes assinaram o termo de consentimento livre e esclarecido, sendo esta pesquisa aprovada pelo Comitê de Ética em Pesquisa da Faculdade Assis Gurgacz, sob o parecer $n^{\circ} 284 / 2007$.

Foram coletados os seguintes dados: pessoais e sociais (nome, idade, renda familiar), hábitos de vida (consumo de álcool, tabagismo, tempo de exposição ao sol) e presença de doenças prévias. Foi realizada também a avaliação antropométrica, dietética e a dosagem de vitamina $\mathrm{D}$ sérica.
Figura 1 - Fluxograma do processo de amostragem

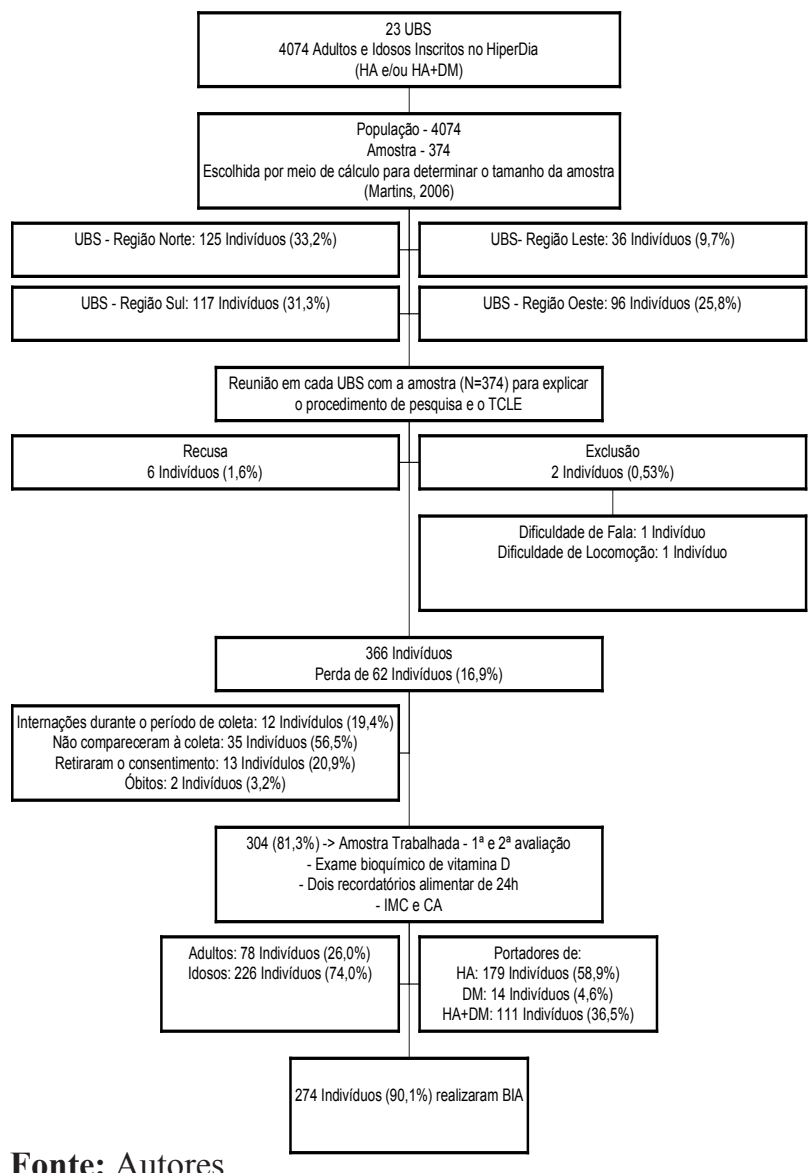

Fonte: Autores

\section{Avaliação antropométrica}

Foram avaliados: peso corpóreo, estatura, circunferência abdominal e porcentagem de gordura corporal. O peso e a altura foram coletados seguindo a metodologia proposta pelo Ministério da Saúde (BRASIL, 2004), utilizando-se balança 
e estadiômetro disponíveis nos postos de saúde e conferindo sua regulagem antes de cada medição. Para avaliação do excesso de peso corporal utilizouse o indicador Índice de Massa Corporal (IMC) (kg/ $\mathrm{m} 2$ ), segundo a proposta da Organização Mundial da Saúde (WORLD HEALTH ORGANIZATION, 1995) para os adultos, que considera sobrepeso IMC $>25 \mathrm{~kg} / \mathrm{m} 2$ e obeso $>30 \mathrm{Kg} / \mathrm{m} 2$, e a Organização Pan-Americana de saúde (ORGANIZAÇÃO PANAMERICANA, 2002) para os idosos (acima de 60 anos), sendo sobrepeso IMC $>28 \mathrm{~kg} / \mathrm{m} 2$ e obeso $>$ $30 \mathrm{~kg} / \mathrm{m} 2$. Para fins de análise estatística, portanto, considerou-se o participante com excesso de peso aqueles com IMC $>25 \mathrm{~kg} / \mathrm{m} 2$ para adultos e $>28 \mathrm{~kg} /$ $\mathrm{m} 2$ para idosos.

Para medida da circunferência abdominal (CA), circundou-se uma fita métrica inelástica no ponto médio entre a crista ilíaca e o rebordo costal inferior dos pacientes no momento da expiração. Considerou-se risco para o desenvolvimento de doença cardiovascular quando se obtiveram valores de $\mathrm{CA}>90 \mathrm{~cm}$ para homens e $>80 \mathrm{~cm}$ para mulheres (ALBERTI et al., 2009)

A porcentagem de gordura corporal foi analisada em 274 pacientes, pois 30 (9,9\%) não seguiram as orientações recomendadas. Esta avaliação foi realizada mediante bioimpedância elétrica (BIA) com auxílio do aparelho Bodystat ${ }^{\circledR}$ modelo 1500 , tetrapolar, frequência de $50 \mathrm{kHz}$, segundo as recomendações de preparo do fabricante. Os pacientes foram classificados como tendo excesso de gordura corporal, segundo Lohman (1992) que considera risco de doenças associadas à obesidade nos valores $>25 \%$ para homens e $>32 \%$ para mulheres.

\section{Avaliação dietética}

Para coleta dos dados de ingestão alimentar foi utilizado o Recordatório Alimentar de 24 horas, que obtém dados de ingestão alimentar do dia que antecede a entrevista, em dois momentos distintos. As informações foram obtidas em medidas caseiras com auxílio de um registro fotográfico (CAVALCANTE; PRIORE; FRANCESCHINI, 2004).

O cálculo da dieta alimentar foi realizado com o auxílio do Programa Dietwin Software ${ }^{\circledR}$ de Análise Nutricional. Foram analisados dados de ingestão de vitamina $\mathrm{D}$ e os seguintes minerais: cálcio, fósforo e magnésio. Os resultados foram comparados seguindo as recomendações do Institute of Medicine of USA (2006).

\section{Avaliação da exposição solar}

O tempo de exposição solar de cada indivíduo foi avaliado de acordo com a quantidade de minutos diário, sendo considerado tempo de exposição adequado $\geq 20$ minutos/dia (MALABANAN; VERONIKIS; HOLICK, 1998).

\section{Dosagem de vitamina $D$ sérica}

Os níveis séricos de 25-hidroxivitamina D (25(OH)D) foram determinados por imunoensaio competitivo de quimioluminescência no equipamento LIASON (DiaSorin, Minesota, USA). Os valores utilizados para categorização dos níveis séricos foram: $<20 \mathrm{ng} / \mathrm{mL}=$ nível deficiente; $\geq 20 \mathrm{e}<$ 30ng/mL = nível bom e $\geq 30 \mathrm{ng} / \mathrm{mL}=$ nível desejável (MALABANAN; VERONIKIS; HOLICK, 1998).

\section{Análise estatística}

Calculou-se a prevalência de inadequação dos minerais: fósforo e magnésio, por meio da Estimated Average Requeriment (EAR) (INSTITUTE OF MEDICINE OF USA, 2006) adotando-se a metodologia proposta por Slater e colaboradores (2004). Devido à assimetria da distribuição da ingestão habitual utilizou-se a transformação logarítmica para análise da prevalência de inadequação. Consideraram-se os valores médios dos dois recordatórios de 24 horas para calcular a ingestão de vitamina $\mathrm{D}$ e cálcio, mas como não há EAR para estes nutrientes não foi realizada a 
inadequação probabilística. Dessa forma, comparouse a média de ingestão de vitamina $\mathrm{D}$ e cálcio com os valores propostos pela Adequate Intake (AI) (INSTITUTE OF MEDICINE OF USA, 2006).

$\mathrm{Na}$ análise descritiva, os dados são apresentados em relação à frequência, média e desvio padrão. Quando necessário, utilizou-se o teste qui quadrado ( $\chi 2)$ com teste exato de Fisher para verificação de associação e aderência. Para comparação de médias ou medianas entre os diferentes grupos utilizou-se do teste de Kruskal-Wallis (não paramétrico). O nível de significância adotado foi $\mathrm{p}<0,05$. O banco de dados foi construído no programa EXCEL® e as análises estatísticas foram realizados nos programas Epi Info ${ }^{\mathrm{TM}}$ e SPSS ${ }^{\circledR}$ for Windows, versão 15.

\section{Resultados}

Encontram-se na Tabela 1 os dados clínicos e demográficos da população estudada. Observa-se que a maioria estudada é de idosos, pertencentes ao gênero feminino, com renda mensal entre um e dois salários mínimos. A deficiência de vitamina $\mathrm{D}$ sérica esteve presente em mais da metade $(52,6 \%)$ dos pacientes estudados e a frequência de HA e DM2 foi de $96,4 \%$ e $46,1 \%$, respectivamente. Verificouse frequência elevada de excesso de peso $(73,4 \%)$, obesidade abdominal $(77,6 \%)$ e de gordura corporal (95,6\%). Em relação à cor da pele $78,6 \%$ eram brancos. Somente $20,8 \%$ dos indivíduos relataram fazer uso de protetor solar e 78,3\% apresentaram baixo tempo de exposição solar. Nenhum indivíduo do estudo fazia uso de suplemento de cálcio e/ou vitamina D.
Tabela 1 - Características clínicas e demográficas dos participantes do Sistema HiperDia, Cascavel, Paraná 2007-2008.

\begin{tabular}{|c|c|c|c|c|}
\hline \multirow[b]{2}{*}{ Variáveis } & \multirow[b]{2}{*}{ Classes } & \multicolumn{2}{|c|}{ Frequência } & \multirow[b]{2}{*}{ IC 95\% } \\
\hline & & & $\%$ & \\
\hline Gênero & Masculino & 82 & 27,0 & $22,1-32,4$ \\
\hline \multirow[t]{3}{*}{ Faixa etária (anos) } & Feminino & 222 & 73,0 & $67,7-77,9$ \\
\hline & Adulto & 67 & 22,0 & $17,6-27,2$ \\
\hline & Idoso & 237 & 78,0 & $72,9-82,5$ \\
\hline \multirow{3}{*}{$\begin{array}{l}\text { Níveis séricos de vitamina D } \\
(\mathrm{ng} / \mathrm{mL})\end{array}$} & Deficiente $(<20)$ & 160 & 52,6 & $46,9-58,4$ \\
\hline & $\operatorname{Bom}(\geq 20 \mathrm{e}<30)$ & 107 & 35,2 & $29,9-40,9$ \\
\hline & Desejável $(\geq 30)$ & 37 & 12,2 & $8,8-16,5$ \\
\hline \multirow{2}{*}{\begin{tabular}{|l|} 
Presença de \\
Diabetes melito
\end{tabular}} & Sim & 125 & 41,1 & $35,5-46,9$ \\
\hline & Não & 179 & 58,9 & $53,1-64,5$ \\
\hline \multirow{7}{*}{$\begin{array}{l}\text { Presença de } \\
\text { Hipertensão arterial } \\
\text { Renda salarial mensal (R\$) }\end{array}$} & Sim & 293 & 96,4 & $93,4-98,1$ \\
\hline & Não & 11 & 3,6 & $1,9-6,6$ \\
\hline & $\leqq 1$ salário & 97 & 31,9 & $26,8-37,5$ \\
\hline & $\geq 1$ salário $\mathrm{e} \leq 2$ salários & 116 & 38,2 & $32,7-43,9$ \\
\hline & $\geq 2$ salários a $\leq 4$ salários & 73 & 24,0 & $19,4-29,3$ \\
\hline & $>4$ salários a $\leq 6$ salários & 15 & 4,9 & $2,9-8,2$ \\
\hline & Acima de 6 salários & 3 & 1,0 & $0,3-3,1$ \\
\hline \multirow[t]{4}{*}{ Estado Nutricional } & Desnutrido & 11 & 3,6 & $1,9-6,6$ \\
\hline & Eutrófico & 70 & 23,0 & $18,5-28,3$ \\
\hline & Sobrepeso & 182 & 59,9 & $54,1-65,4$ \\
\hline & Obeso & 41 & 13,5 & $10,0-18,0$ \\
\hline \multirow{3}{*}{$\begin{array}{l}\text { Aumento da circunferência } \\
\text { abdominal }(\mathrm{cm})\end{array}$} & Com risco & 236 & 77,6 & $72,5-82,2$ \\
\hline & Sem risco & 68 & 22,4 & $17,9-27,6$ \\
\hline & & & & $92,5-97,7$ \\
\hline \multirow{2}{*}{ Excesso de gordura Corporal } & Sim & 262 & 95,6 & $2,3-7,5$ \\
\hline & Não & 12 & 4,4 & $73,6-83,1$ \\
\hline \multirow{2}{*}{ Cor da pele } & Brancos e amarelos & 239 & 78,6 & $17-26,5$ \\
\hline & Pretos e pardos & 65 & 21,4 & $16,2-25,9$ \\
\hline \multirow[t]{2}{*}{ Uso de protetor solar } & $\underline{\text { Sim }}$ & 60 & 20,8 & $74,1-83,8$ \\
\hline & Não & 229 & 79,2 & $17,3-26,9$ \\
\hline \multirow[t]{2}{*}{ Exposição solar } & Adequada ( $>20$ minutos $/$ dia) & 66 & 21,7 & $73,2-82,8$ \\
\hline & Inadequada $(<20 \mathrm{minutos} / \mathrm{dia})$ & 238 & 78,3 & \\
\hline
\end{tabular}

IC: Intervalo de confiança

Fonte: Autores.

$\mathrm{O}$ inquérito alimentar evidenciou baixo consumo de cálcio e vitamina $\mathrm{D}$ e alto consumo de fósforo e magnésio (Tabela 2). Entretanto, apesar dos pacientes referirem consumo elevado de fósforo e magnésio, eles mantinham ingestão inadequada destes nutrientes segundo o Institute of Medicine of USA (2006) (Tabela 2). A prevalência de inadequação foi de $100 \%$ para o fósforo e de $81,8 \%$ para o magnésio nos indivíduos do gênero masculino e de $92,7 \%$ e $68,6 \%$, respectivamente, no feminino. Não foi possível estimar a prevalência de inadequação no consumo de vitamina D e cálcio, pois esses nutrientes não possuem EAR. 
Confrontando os dados de ingestão média de cálcio com a recomendação proposta pela $\mathrm{AI}$, apenas 8 $(10,1 \%)$ homens e $8(3,9 \%)$ mulheres com idade acima ou igual a 51 anos ingeriam a recomendação adequada para a idade, ou seja, $\geq 1200 \mathrm{mg}$ de cálcio/ dia. Ressalta-se que, em ambos os gêneros na faixa etária de 31 a 50 anos, nenhum indivíduo ingeria as quantidades de cálcio recomendadas. Quanto à ingestão de vitamina $\mathrm{D}$, apenas uma mulher ingeria a recomendação preconizada, isto é $5 \mu \mathrm{g} / \mathrm{dia}$.

Tabela 2 - Ingestão de nutrientes e prevalência de inadequação do consumo de fósforo, magnésio, cálcio e vitamina D dos participantes do Sistema HiperDia, Cascavel, Paraná, 2007-2008.

\begin{tabular}{|c|c|c|c|c|c|c|}
\hline & & \multicolumn{3}{|c|}{ Homem } & \multirow{2}{*}{\begin{tabular}{|c|} 
Mulher \\
RN
\end{tabular}} & \multirow[b]{2}{*}{ Média $\pm D P$} \\
\hline & & RN & Média土DP & Inadequação & & \\
\hline$P(m g)^{a}$ & $\begin{array}{c}\text { Todos } \\
\text { participantes }\end{array}$ & 580 & $1048 \pm 500,0$ & $100 \%$ & 580 & $1060 \pm 510,9$ \\
\hline $\mathrm{Mg}(\mathrm{mg})^{\text {a }}$ & $\begin{array}{c}\text { Todos } \\
\text { participantes }\end{array}$ & 350 & $404,6 \pm 209,01$ & $81,8 \%$ & 265 & $407,2 \pm 208,8$ \\
\hline \multirow{2}{*}{$\mathrm{Ca}(\mathrm{mg})^{\mathrm{b}}$} & $31-50$ anos & 1000 & $481,4 \pm 275,2$ & - & 1000 & $457,1 \pm 285,1$ \\
\hline & $\geq 51$ anos & 1200 & $644,3 \pm 384,7$ & - & 1200 & $579,9 \pm 303,2$ \\
\hline \multirow{3}{*}{ Vit D (ug) ${ }^{b}$} & $31-50$ anos & 5 & $2,18 \pm 2,36$ & - & 5 & $1,72 \pm 1,56$ \\
\hline & $51-70$ anos & 10 & $2,70 \pm 1,84$ & & 10 & $2,98 \pm 2,12$ \\
\hline & $\geq 71$ anos & 15 & $3,13 \pm 2,09$ & - & 15 & $2,57 \pm 2,12$ \\
\hline
\end{tabular}

RN: Recomendação nutricional; DP: desvio padrão; a: Utilizada EAR - Estimated Average Requeriment; b: Utilizada a AI - Adequate Intake - Não pode ser avaliada a prevalência de inadequação; $\mathrm{P}$ : fósforo; $\mathrm{Mg}$ : magnésio; Ca: cálcio; Vit D: vitamina D.

Fonte: Autores

Com relação aos dados demográficos e clínicos dos pacientes (Tabela 3), observou-se associação significativa entre os dados de circunferência abdominal aumentada $(\mathrm{p}<0,05)$, exposição solar inadequada $(p<0,001)$ e hipertensão arterial $(\mathrm{p}=0,0006)$ com os diferentes níveis séricos de vitamina $\mathrm{D}$, sendo que estes fatores, quando presentes, apresentaram menor frequência no grupo de pacientes com níveis séricos desejáveis de vitamina D. Os demais dados estudados não apresentaram associação significativa com os níveis séricos de vitamina $\mathrm{D}$.
Tabela 3 - Associação entre os dados demográficos e clínicos de indivíduos participantes do sistema HiperDia de acordo com os níveis séricos de vitamina D, Cascavel, Paraná, 2007-2008.

\begin{tabular}{|c|c|c|c|c|c|c|c|c|}
\hline \multirow{3}{*}{ Variáveis } & \multirow{3}{*}{ Classes } & \multicolumn{6}{|c|}{ Níveis séricos de vitamina $D^{3}$} & \multirow{3}{*}{$\mathbf{P}$} \\
\hline & & \multicolumn{2}{|c|}{$\begin{array}{c}\text { Deficiente } \\
(n=160)\end{array}$} & \multicolumn{2}{|c|}{$\begin{array}{c}\text { Suficiente } \\
(\mathrm{n}=107)\end{array}$} & \multicolumn{2}{|c|}{$\begin{array}{c}\text { Desejável } \\
(\mathrm{n}=37)\end{array}$} & \\
\hline & & $\mathbf{n}$ & $\%$ & $\mathbf{n}$ & $\%$ & $\mathbf{n}$ & $\%$ & \\
\hline \multirow{2}{*}{ Gênero } & Masculino & 35 & 21,9 & 34 & 31,8 & 13 & 35,1 & \multirow{2}{*}{0,10} \\
\hline & Feminino & 125 & 78,1 & 73 & 68,2 & 24 & 64,9 & \\
\hline \multirow{2}{*}{ Faixa etária (anos) } & Adulto & 32 & 20,0 & 25 & 23,4 & 10 & 27,0 & \multirow{2}{*}{0,59} \\
\hline & Idoso & 128 & 80,0 & 82 & 76,6 & 27 & 73,0 & \\
\hline \multirow{2}{*}{ Tabagismo } & Sim & 11 & 7,0 & 6 & 5,7 & 5 & 13,5 & \multirow{2}{*}{0,28} \\
\hline & Não & 146 & 93,0 & 100 & 94,3 & 32 & 86,5 & \\
\hline \multirow{2}{*}{ Consumo de álcool } & Sim & 28 & 17,5 & 17 & 15,9 & 3 & 8,1 & \multirow{2}{*}{0,37} \\
\hline & Não & 132 & 82,5 & 90 & 84,1 & 34 & 91,9 & \\
\hline \multirow{2}{*}{ Excesso de peso $0^{b}$} & Sim & 116 & 72,5 & 84 & 78,5 & 23 & 62,2 & \multirow{2}{*}{0,14} \\
\hline & Não & 44 & 27,5 & 23 & 21,5 & 14 & 37,8 & \\
\hline \multirow{2}{*}{$\begin{array}{l}\text { Excesso de gordura } \\
\text { Corporal }{ }^{\circ}\end{array}$} & Sim & 153 & 95,3 & 103 & 95,8 & 36 & 96,7 & \multirow{2}{*}{0,89} \\
\hline & Não & 7 & 4,7 & 4 & 4,2 & 1 & 3,3 & \\
\hline \multirow{2}{*}{$\begin{array}{l}\text { Aumento da circunferência } \\
\text { abdominal }^{d}\end{array}$} & Sim & 126 & 78,8 & 87 & 81,3 & 23 & 62,2 & \multirow{2}{*}{$<0,05$} \\
\hline & Não & 34 & 21,3 & 20 & 18,7 & 14 & 37,8 & \\
\hline \multirow{2}{*}{ Diabetes Melito } & Sim & 70 & 43,8 & 39 & 36,4 & 16 & 43,2 & \multirow{2}{*}{0,48} \\
\hline & Não & 90 & 56,3 & 68 & 63,6 & 21 & 56,8 & \\
\hline \multirow{2}{*}{ Hipertensão Arterial } & Sim & 159 & 99,4 & 102 & 95,3 & 32 & 86,5 & \multirow{2}{*}{0,0006} \\
\hline & Não & 1 & 0,6 & 5 & 4,7 & 5 & 13,5 & \\
\hline \multirow{2}{*}{ Tempo de exposição ao sol } & Inadequado & 121 & 75,6 & 69 & 64,5 & 16 & 43,2 & \multirow{2}{*}{$<0,00$} \\
\hline & Adequado & 39 & 24,4 & 38 & 35,5 & 21 & 56,8 & \\
\hline \multirow{2}{*}{ Protetor solar } & Sim & 25 & 16,3 & 27 & 26,5 & 8 & 23,5 & 014 \\
\hline & Não & 128 & 83,7 & 75 & 73,5 & 26 & 76,5 & \\
\hline & Sim & 80 & 50,0 & 64 & 59,3 & 24 & 64,9 & \\
\hline Aurvace & \begin{tabular}{|l|} 
Não \\
\end{tabular} & 80 & 50,0 & 43 & 40,2 & 13 & 35,1 & 0,15 \\
\hline Medicamento de uso & Sim & 154 & 96,3 & 103 & 96,3 & 37 & 100,0 & 0.15 \\
\hline contínuo & \begin{tabular}{|l|l} 
Não \\
\end{tabular} & 6 & 3,8 & 4 & 3,7 & 0 & 0,0 & 0,15 \\
\hline Osteoporose & Sim & 28 & 17,5 & 12 & 11,3 & 5 & 13,5 & 0,37 \\
\hline & Não & 132 & 82,5 & 94 & 88,7 & 32 & 86,5 & \\
\hline & $\begin{array}{l}\text { Brancose } \\
\text { amarelos }\end{array}$ & 132 & 82,5 & 83 & 77,6 & 24 & 64,9 & \\
\hline or da ple & $\begin{array}{l}\text { Pretos e } \\
\text { pardos }\end{array}$ & 28 & 17,5 & 24 & 22,4 & 13 & 35,1 & 0,00 \\
\hline
\end{tabular}

Teste Qui-quadrado. a:Classificação: nível Deficiente < 20ng/mL; Suficiente $\geq 20$ e $<30 \mathrm{ng} / \mathrm{mL}$; nível Desejável $\geq 30 \mathrm{ng} / \mathrm{mL}$; b: Índice de massa corporal $>25 \mathrm{Kg} / \mathrm{m} 2$ para adultos e $>28 \mathrm{Kg} / \mathrm{m} 2$ para idosos; c: Avaliado por meio de bioimpedância elétrica: \% gordura corporal $>32 \%$ para mulheres e $>25 \%$ para homens; d: Aumento da circunferência abdominal: $>102 \mathrm{~cm}$ para homens e $>88 \mathrm{~cm}$ para mulheres; e: Tempo de exposição ao sol: adequado $>20$ minutos ao dia e inadequado $<20$ minutos ao dia.

Fonte: Autores.

Analisando os dados de IMC, CA, porcentagem de gordura corporal, ingestão de cálcio, vitamina $\mathrm{D}$, fósforo, magnésio e tempo de exposição ao sol com os diferentes níveis séricos de vitamina D (Tabela 4), observou-se que pacientes com níveis séricos desejáveis de vitamina $\mathrm{D}$ apresentavam 
significativamente menores valores de IMC $(p<0,03)$ e $C A(p<0,01)$ e maior tempo de exposição solar $(p=0,01)$ quando comparados aos pacientes com níveis séricos de vitamina $\mathrm{D}$ classificados como deficientes e bons. Não houve associação entre níveis séricos de vitamina $\mathrm{D}$ e ingestão de cálcio, fósforo e magnésio.

Tabela 4 - Dados clínicos e laboratoriais dos participantes do Sistema HiperDia, Cascavel, Paraná, 2007-2008.

\begin{tabular}{|c|c|c|c|c|}
\hline & $\begin{array}{c}\text { Deficiente }^{3} \\
(\mathbf{n}=160)\end{array}$ & $\begin{array}{c}\text { Suficiente }^{\text {a }} \\
(\mathbf{n}=107)\end{array}$ & $\begin{array}{c}\text { Dese jável a }^{{ }^{2}} \\
\quad(\mathbf{n}=37)\end{array}$ & $\mathrm{p}$ \\
\hline $\operatorname{IMC}\left(\mathrm{Kg} / \mathrm{m}^{2}\right)$ & $\begin{array}{c}29,1 \\
(17,2-54,7)\end{array}$ & $\begin{array}{c}29,6 \\
(19,2-29,6)\end{array}$ & $\begin{array}{c}27,9 \\
(17,4-35,9)\end{array}$ & 0,03 \\
\hline $\mathrm{CA}(\mathrm{cm})$ & $\begin{array}{c}101 \\
(59-160)\end{array}$ & $\begin{array}{c}104 \\
(66-138)\end{array}$ & $\begin{array}{c}97 \\
(68-119)\end{array}$ & $<0,01$ \\
\hline GC (\%) & $\begin{array}{c}41,7 \\
(23,2-58,1)\end{array}$ & $\begin{array}{c}41,8 \\
(18,5-56,5)\end{array}$ & $\begin{array}{c}40,7 \\
(15,6-51,1)\end{array}$ & 0,36 \\
\hline $\mathrm{Ca}(\mathrm{mg})$ & $\begin{array}{c}549,2 \\
(103,4-2017,4)\end{array}$ & $\begin{array}{c}502,4 \\
(71,2-1856,2)\end{array}$ & $\begin{array}{c}527,9 \\
(141,7-1442,5)\end{array}$ & 0,13 \\
\hline Vit D (ug) & $\begin{array}{c}1,84 \\
(0-8,05)\end{array}$ & $\begin{array}{c}2,39 \\
(0-16,5)\end{array}$ & $\begin{array}{c}2,53 \\
(0-7,45)\end{array}$ & 0,16 \\
\hline $\mathrm{P}(\mathrm{mg})$ & $\begin{array}{c}993,7 \\
(249,7-3644,6)\end{array}$ & $\begin{array}{c}975,7 \\
(306,0-2805,4)\end{array}$ & $\begin{array}{c}1054,6 \\
(323,6-2311,4)\end{array}$ & 0,42 \\
\hline $\mathrm{Mg}(\mathrm{mg})$ & $\begin{array}{c}378,4 \\
(44,8-1165,7)\end{array}$ & $\begin{array}{c}401,4 \\
(81,7-950,5)\end{array}$ & $\begin{array}{c}373,6 \\
(51,0-1213,6)\end{array}$ & 0,64 \\
\hline $\begin{array}{l}\text { Exposição ao } \\
\text { sol (min/dia) }\end{array}$ & $\begin{array}{c}9,3 \\
(0-56,0)\end{array}$ & $\begin{array}{c}12 \\
(0-80)\end{array}$ & $\begin{array}{c}28 \\
(0-80)\end{array}$ & 0,01 \\
\hline
\end{tabular}

Teste de Kruskal-Wallis (distribuição não-normal); a: Classificação: nível Deficiente $<20 \mathrm{ng} / \mathrm{ml}$; Nível bom $\geq$ 20 e <30 ng/ml; nível Desejável $\geq 30 \mathrm{ng} / \mathrm{mL}$. IMC: índice de massa corpórea; CA: circunferência abdominal; GC: gordura corporal; Vit $\mathrm{D}$ : vitamina $\mathrm{D}$; $\mathrm{Ca}$ : cálcio; $\mathrm{P}$ : fósforo; Mg: magnésio.

Fonte: Autores.

\section{Discussão}

Os principais achados do presente trabalho foram a frequência elevada de hipovitaminose $\mathrm{D}$ em pacientes com HA e DM2, a associação inversa entre os níveis séricos desejáveis de vitamina $\mathrm{D}$ e hipertensão arterial e com a circunferência abdominal e a associação direta entre tempo adequado de exposição solar e níveis séricos desejáveis de vitamina $\mathrm{D}$.

No presente trabalho, a deficiência de vitamina $\mathrm{D}$, definida como nível sérico de $25(\mathrm{OH}) \mathrm{D}<20 \mathrm{ng} /$
$\mathrm{mL}$, foi detectada em 52,6\% dos pacientes do Sistema HiperDia. Não é de nosso conhecimento, até o presente momento, estudo populacional sobre a prevalência de deficiência de vitamina $\mathrm{D}$ em nível nacional. Outros trabalhos apresentaram prevalências menores (CANTO-COSTA; KUNII; HAUACHE, 2006; SILVA et al., 2008) que as encontradas no presente estudo com exceção de Saraiva et al. (2007) que avaliaram pacientes institucionalizados e encontraram prevalência de $71,2 \%$ de deficiência de vitamina D. Utilizando ponto de corte diferenciado (25(OH)D $<30 \mathrm{ng} / \mathrm{ml})$, Lips et al. (2006) encontraram prevalência de $63,9 \%$ de hipovitaminose D em pesquisa que envolveu 18 países do continente Europeu, Asiático, Americano e da Oceania.

Atualmente, a deficiência de vitamina $\mathrm{D}$ tem sido considerada um problema de saúde pública mundial, em razão de suas implicações no desenvolvimento de diversas DCNT, além do papel já conhecido na integridade óssea (MITHAL et al., 2009). A estimativa é que a insuficiência de vitamina $D$ já tenha atingido um bilhão de indivíduos ao redor do mundo (KIMBALL; FULEIHAN; VIETH, 2008).

Segundo a classificação do estado nutricional por meio do IMC, verificou-se frequência de 73,4\% de excesso de peso (sobrepeso e obesidade) nos pacientes do Sistema HiperDia. Como era esperada, em população portadora de hipertensão e diabetes, esta frequência foi superior aos dados da Pesquisa de Orçamento Familiar (POF) (IBGE, 2010), realizada em 2008-2009, que apresentou 63,8\% de excesso de peso na população brasileira.

No presente trabalho verificou-se percentual de gordura corporal extremamente elevado $(95,6 \%)$, sendo este dado superior ao excesso de peso $(73,4 \%)$. Isto pode ter ocorrido pela ausência, até o momento, de uma classificação de gordura corporal específica para idosos, que devido ao processo de envelhecimento, apresenta aumento do tecido adiposo. Ressalta-se que o excesso de peso é menos identificado pelo IMC do que pelo percentual de gordura corpórea (MCGILL et al., 2008). Há controvérsia sobre a pressuposição 
de que o IMC mede com a mesma precisão o teor de gordura corpórea em todas as faixas etárias, notadamente no envelhecimento (BEDOGNI et al., 2001; MOVSESYAN et al., 2003) e, dessa forma, há a necessidade deste indicador estar associado a outros índices antropométricos, como por exemplo, o percentual de gordura.

Encontrou-se alto percentual (77,6\%) de pacientes com obesidade abdominal. Sabe-se que a CA é indicadora da gordura abdominal e é considerada preditora de doenças metabólicas e cardiovasculares, dentre essas, a hipertensão arterial e o diabetes melito (STERNFELD, 2002). Indivíduos com obesidade apresentam aumento na morbimortalidade decorrente das DCNT, tais como doença cardíaca coronariana, DM2 e HA (CERCATO et al., 2004). Ressalta-se que todos os participantes avaliados no presente estudo eram acometidos pelas duas últimas doenças, reforçando, dessa forma, a importância do conhecimento sobre a composição corpórea do indivíduo não somente como medida preventiva de DCNT, mas, também, em decorrência de suas potenciais consequências deletérias.

O consumo de micronutrientes entre os grupos de adultos e idosos foi bastante semelhante, mostrando hábitos alimentares inadequados. Houve alta prevalência de inadequação dos minerais fósforo e magnésio, assim como o consumo de vitamina D e cálcio foi insuficiente. Sabe-se que a vitamina D tem relação direta com o metabolismo do cálcio, fósforo e magnésio, portanto sua diminuição, tanto dietética como sérica, pode resultar em desequilíbrio metabólico, principalmente na saúde óssea (COZZOLINO, 2005). Assim, dietas contendo quantidades insuficientes desses micronutrientes podem levar à baixa densidade óssea, levando a implicações como risco de osteoporose ao longo da vida e ao desenvolvimento de doenças metabólicas, como citado anteriormente.

Nota-se, no presente estudo, que não houve associação estatística significativa entre a ingestão de vitamina $\mathrm{D}$ e os diferentes níveis séricos de $25(\mathrm{OH})$
$\mathrm{D}$, isto pode ser atribuído ao fato de que a ingestão média de vitamina $\mathrm{D}$ de todos os indivíduos $(97,3 \%)$ avaliados encontrou-se bem abaixo da recomendação.

Observou-se menor frequência de obesidade abdominal e menor média de CA nos pacientes com níveis desejáveis de vitamina D. Estes dados corroboram com as evidências que apontam que baixos níveis séricos de vitamina D estão relacionados com a alteração da função celular em vários tecidos, incluindo o pâncreas endócrino, que está envolvido no desenvolvimento da obesidade, principalmente abdominal, DM2 (MCGILL et al., 2008), síndrome metabólica (SM) e DCV (HOLICK; CHEN, 2008). Anne-Thea McGill e colaboradores (2008) correlacionaram a vitamina D com gordura corporal, parâmetros da SM e hemoglobina glicosilada (HbA1c) em um estudo envolvendo 250 pacientes adultos com idade superior a 18 anos com excesso de peso e obesidade (IMC entre 28 a $50 \mathrm{~kg} / \mathrm{m} 2$ ) de diferentes etnias em Auckland na Nova Zelândia. Os resultados mostraram que os níveis séricos de vitamina D foram inversamente relacionados com peso, IMC e marcadores de DM2 (CA e aumento da HbA1c). Os autores também verificaram após realização de regressão multivariada, que houve redução de $0,74 \mathrm{nmo} / \mathrm{L}(\mathrm{p}=0,0002)$ na vitamina $\mathrm{D}$ a cada $1 \mathrm{~kg} / \mathrm{m} 2$ de aumento no IMC e uma redução de $0,2 \mathrm{nmol} / \mathrm{L}(\mathrm{p}=0,01)$ a cada centímetro de aumento na cintura, o que explica aproximadamente 3\% na variação de vitamina $\mathrm{D}$ independentemente do gênero, idade, etnia e estação climática.

No presente estudo, o grupo de indivíduos com níveis desejáveis de vitamina $\mathrm{D}$ apresentaram menor média de IMC. Hyppönen e Power (1998) avaliaram a inter-relação entre vitamina $\mathrm{D}$, composição corporal e homeostase da glicose mensurada em 7.198 sujeitos caucasianos britânicos nascidos em 1958. Os resultados mostraram que a concentração de vitamina $\mathrm{D}$ foi $<75 \mathrm{nmol} / \mathrm{L}$ em $80 \%$ dos sujeitos obesos (IMC $\geq 30 \mathrm{~kg} / \mathrm{m} 2$ ) e de $68 \%$ nos demais ( $\mathrm{P}$ $<0.0001)$. 
A associação entre hipovitaminose $\mathrm{D}$ e obesidade ocorre devido a diversos mecanismos; 10) a vitamina $\mathrm{D}$ pode provocar resistência à ação da insulina e contribuir em vários outros processos metabólicos no tecido adiposo e muscular; 2o) a vitamina D se encontra em maior quantidade no tecido adiposo do que na circulação; 3o) as pessoas obesas, muitas vezes, podem sentir-se constrangidas com a exposição do seu corpo, minimizando assim a exposição ao sol, com consequente diminuição da síntese de vitamina D (MCGILL et al., 2008).

Snijder e colaboradores (2005) ressaltam que a gordura corporal total está inversamente associada com níveis séricos de vitamina $\mathrm{D}$ e um maior estoque de gordura nos tecidos adiposos é uma explicação plausível para elevadas taxas de deficiência em indivíduos obesos.

Encontrou-se associação entre tempo adequado de exposição solar e níveis séricos desejáveis de vitamina D. Esse dado, somado a ausência de associação entre ingestão dietética e níveis séricos de vitamina $\mathrm{D}$, nos permite inferir que a exposição solar foi o fator que mais influenciou os níveis séricos de vitamina $\mathrm{D}$ nesta população e não a ingestão dietética desta vitamina, visto que sua ingestão foi insuficiente.

Os achados do presente estudo, mostrando níveis maiores de 25(OH)D em indivíduos com exposição solar adequada ( $\geq 20$ minutos/dia) (MALABANAN; VERONIKIS; HOLICK, 1998) corrobora com dados da literatura, ressaltando a importância desse fator como prevenção de deficiência de vitamina D.

Até recentemente, acreditava-se que a discussão sobre a falta de vitamina $\mathrm{D}$ era desnecessária no Brasil, haja vista ser este país tropical que recebe luz solar abundante, sendo a maior parte da vitamina D sintetizada com a sua ajuda. Contudo, em nossa sociedade, a ingestão de vitamina D voltou a ter importância devido à pouca exposição à luz solar e outros fatores ainda não bem estabelecidos (PREMAOR; FURLANETTO, 2006).

Embora os estudos acerca da importância na diminuição da incidência de DM2 (PITTAS et al.,
2006, 2007) e HA (PILZ; TOMASCHITZ, 2010) em populações com níveis desejáveis de vitamina D sejam mais consistentes do que estudos com suplementação com vitamina D, Witham, Nadir e Struthers (2009) verificaram em meta-análise que houve diminuição significativa da pressão diastólica em pacientes hipertensos com uso de vitamina $\mathrm{D}$ e cálcio. Com relação à suplementação de vitamina $\mathrm{D}$ em pacientes com DM2, revisão sistemática recente não verificou melhora no metabolismo de carboidratos em pacientes com diagnóstico estabelecido de DM2, embora haja relatos de diminuição da resistência à insulina em pacientes com intolerância à glicose (MITRI; MURARU; PITTAS, 2011).

Em conclusão, o presente trabalho permitiu verificar a alta frequência de hipovitaminose $\mathrm{D}$ e sua associação com a diminuição de exposição solar, com adiposidade e com a hipertensão arterial. Estudos randomizados e controlados em pacientes com HA e DM2 e deficiência de Vitamina D são necessários para verificar a melhora clínica verificada em alguns estudos após introdução de suplementação com vitamina $\mathrm{D}$ nestes pacientes.

\section{Agradecimentos}

Ao laboratório Álvaro e ao Dr. Fabiano Sandrini pelas análises bioquímicas, à Faculdade Assis Gurgacz pelo apoio financeiro e à Secretaria Municipal de Saúde de Cascavel, pela permissão da realização desta pesquisa.

\section{Referências}

ALBERTI, K. G.; ECKEL, R. H.; GRUNDY, S. M.; ZIMMET, P. Z.; CLEEMAN, J. I.; DONATO, K. A.; FRUCHART, J. C.; JAMES, W. P.; LORIA, C. M.; SMITH, S. C. Harmonizing the metabolic syndrome: a joint interim statement of the International Diabetes Federation Task Force on Epidemiology and Prevention; National Heart, Lung, and Blood Institute; American Heart Association; World Heart Federation; International. Circulation, Dallas, v. 120, n. 16, p. 1640-1645, 20 out. 2009. 
BEDOGNI, G.; PIETROBELLI, A.; HEYMSFIELD, S.B.; BORGHI, A.; MANZIERI, A.M.; MORINI, P.; BATTISTINI, N.; SALVIOLI G. Is body mass index a measure of adiposity in elderly women? Obesity research, Modena, v. 9, n. 1, p. 17-20, jan. 2001.

BRASIL. Ministério da Saúde. Datasus: informações de saúde. Disponível em: <www.datasus.gov.br>. Acesso em: 10 ago. 2008.

BRASIL. Ministério da Saúde. Vigilância alimentar e nutricional - SISVAN: orientações básicas para a coleta, processamento, análise de dados e informações em serviços de saúde. Brasília, Ministério da Saúde, 2004.

CANTO-COSTA, M. H. S.; KUNII, I.; HAUACHE, O. M. Body fat and cholecalciferol supplementation in elderly homebound individuals. Brazilian Journal of Medical and Biological Research, São Paulo, v. 39, n. 1, p. 91-98, jan. 2006.

CAVAlCANTE, A. A. M.; PRIORE, S. E.; FRANCESCHINI, S. C. C. Estudos de consumo alimentar: aspectos metodológicos gerais e o seu emprego na avaliação de crianças e adolescentes. Revista Brasileira de Saúde Materno Infantil, São Paulo, v. 4, n. 3, p. 229-240, 2004.

CERCATO, C.; MANCINI, M. C.; ARGUELLO, A. M. C.; PASSOS, V. Q.; VILLARES, S. M. F.; HALPERN, A. Systemic hypertension, diabetes mellitus, and dyslipidemia in relation to body mass index: evaluation of a Brazilian population. Revista do Hospital das Clínicas, São Paulo, v. 59, n. 3, p. 113-118, 2004.

COZZOLINO, S. M. F. Biodisponibilidade de nutrientes. Barueri: Manole, 2005.

HOLICK, M. F.; CHEN, T. C. Vitamin D deficiency: a worldwide problem with health consequences. The American journal of clinical nutrition, London, v. 87, n. 4, p. 1080S-1086S, abr. 2008.

HYPPÖNEN, E.; POWER, C. Vitamin D status and glucose homeostasis in the 1958 British birth cohort: the role of obesity. Diabetes care, Londres, v. 29, n. 10, p. 2244-2246, out. 2006.
IBGE. Pesquisa de orçamento familiares 2008-2009: Antropometria e estado nutricional de crianças, adolescentes e adultos no Brasil. Rio de Janeiro, 2010.

INSTITUTE OF MEDICINE OF USA. National Research Council. Food and Nutrition Board. Dietary references intakes: the essential guide to nutrient requirements. Washington: National Academy Press, 2006

KIMBALL, S.; FULEIHAN, G. E.-H.; VIETH, R. Vitamin D: a growing perspective. Critical reviews in clinical laboratory sciences, London, v. 45, n. 4, p. 339-414, jan. 2008.

LIPS, P.; HOSKING, D.; LIPPUNER， K.; NORQUIST, J. M.; WEHREN, L.; MAALOUF, G.; RAGI-EIS, S.; CHANDLER, J. The prevalence of vitamin D inadequacy amongst women with osteoporosis: an international epidemiological investigation. Journal of internal medicine, Oxford, v. 260 , n. 3 , p. $245-54$, set. 2006.

LOHMAN, T. G. Advances in body composition assessment. Champaign: Human Kinetics Publishers, 1992.

MALABANAN, A.; VERONIKIS, I. E.; HOLICK, M. F. Redefining vitamin D insufficiency. Lancet, London, v. 351, n. 9105, p. 805-806, 14 mar. 1998.

MCGILL, A. T.; STEWART, J. M.; LITHANDER, F. E.; STRIK, C. M.; POPPITT, S. D. Relationships of low serum vitamin D3 with anthropometry and markers of the metabolic syndrome and diabetes in overweight and obesity. Nutrition Journal, London, v. 7, p. 4 , jan. 2008 .

MITHAL, A.; WAHL, D. A.; BONJOUR, J. P.; BURCKHARDT, P.; DAWSON-HUGHES, B.; EISMAN, J. A.; EL-HAJJ FULEIHAN, G.; JOSSE, R. G.; LIPS, P.; MORALES-TORRES, J. Global vitamin D status and determinants of hypovitaminosis D. Osteoporosis International, London, v. 20, n. 11, p. 1807-1820, nov. 2009.

MITRI, J.; MURARU, M. D.; PITTAS, A. G. Vitamin $\mathrm{D}$ and type 2 diabetes: a systematic review. European journal of Clinical Nutrition, London, v. 65, n. 9, p. 1005-1015, set. 2011. 
MOVSESYAN, L.; TANKÓ, L.B.; LARSEN, P.J.; CHRISTIANSEN, C.; SVENDSEN, O.L. Variations in percentage of body fat within different BMI groups in young, middle-aged and old women. Clinical Physiology and Functional Imaging, Oxford, v. 23, n. 3, p. 130-3, maio 2003.

\section{ORGANIZAÇÃO PAN-AMERICANA.} Informe preliminar da $36^{\text {a }}$ Reunión del Comité Asesor de Investigaciones en Salud - Encuesta Multicéntrica - Salud Bienestar y Envejecimento (SABE) en América Latina y el Caribe, 2002.

PETERLIK, M.; CROSS, H. S. Vitamin D and calcium deficits predispose for multiple chronic diseases. European Journal of Clinical Investigation, Barcelona, v. 35, n. 5, p. 290-304, maio 2005.

PILZ, S.; TOMASCHITZ, A. Role of vitamin $\mathrm{D}$ in arterial hypertension. Expert review of Cardiovascular Therapy, London, v. 8, n. 11, p. 1599-1608, nov. 2010.

PITTAS, A. G.; DAWSON-HUGHES, B.; LI, T.; VAN DAM, R. M.; WILLETT, W. C.; MANSON, J. E.; HU, F. B. Vitamin D and calcium intake in relation to type 2 diabetes in women. Diabetes care, Alexandria, v. 29, n. 3, p. 650-656, mar. 2006.

PITTAS, A.G;; LAU, J.; HU, F.B.; DAWSONHUGHES, B. The role of vitamin D and calcium in type 2 diabetes. A systematic review and metaanalysis. The Journal of Clinical Endocrinology and Metabolism, Washington, v. 92, n. 6, p. 2017-29, jun. 2007.

PREMAOR, M. O.; FURLANETTO, T. W. Hipovitaminose D em adultos: entendendo melhor a apresentação de uma velha doença. Arquivos Brasileiros de Endocrinologia \& Metabologia, Botucatu, v. 50, n. 1, p. 25-37, fev. 2006.

PRENTICE, A.; GOLDBERG, G. R.; SCHOENMAKERS, I. Vitamin D across the lifecycle: physiology and biomarkers. The American Journal of Clinical Nutrition, Houston, v. 88, n. 2, p. 500S-506S, ago. 2008.
SARAIVA, G. L.; CENDOROGLO, M. S.; RAMOS, L. R.; ARAÚJO, L. M. Q.; VIEIRA, J. G. H.; MAEDA, S. S.; BORBA, V. Z.C; KUNII, I.; HAYASHI, L. F.; LAZARETTI-CASTRO, M. Prevalência da deficiência, insuficiência de vitamina $\mathrm{D}$ e hiperparatiroidismo secundário em idosos institucionalizados e moradores na comunidade da cidade de São Paulo, Brasil. Arquivos Brasileiros de Endocrinologia \& Metabologia, Botucatu, v. 51, n. 3, p. 437-442, abr. 2007.

SILVA, B. C. C; CAMARGOS, B. M.; FUJII, J. B.; DIAS, E. P.; SOARES, M. M. S. Prevalência de deficiência e insuficiência de vitamina $D$ e sua correlação com PTH, marcadores de remodelação óssea e densidade mineral óssea, em pacientes ambulatoriais. Arquivos Brasileiros de Endocrinologia \& Metabologia, Botocuatu, v. 52, n. 3, p. 482-488, abr. 2008.

SNIJDER, M. B.; VAN DAM, R. M.; VISSER, M.; DEEG, D. J.; DEKKER, J. M.; BOUTER, L. M.; SEIDELL, J. C.; LIPS, P. Adiposity in relation to vitamin D status and parathyroid hormone levels: a population-based study in older men and women. The Journal of Clinical Endocrinology and Metabolism, Washington v. 90, n. 7, p. 4119-4123, jul. 2005.

SOCIEDADE BRASILEIRA DE CARDIOLOGIA. IV Diretrizes Brasileiras de Hipertensão. Arquivos Brasileiros de Cardiologia, São Paulo, v. 95, p.1-55, Rio de Janeiro, 2010.

STERNFELD, B. Associations of Body Composition with Physical Performance and Self-reported Functional Limitation in Elderly Men and Women. American Journal of Epidemiology, London, v. 156, n. 2, p. 110-121, 15 jul. 2002.

WITHAM, M. D.; NADIR, M. A.; STRUTHERS, A. D. Effect of vitamin D on blood pressure: a systematic review and meta-analysis. Journal of hypertension, Milan, v. 27, n. 10, p. 1948-54, out. 2009.

WORLD HEALTH ORGANIZATION. Physical status: the use and interpretation of anthropometry. Geneva: World Health Organization Technical Report Series, 1995.

Recebido em: 31 jul. 2014 Aceito em: 12 nov. 2014. 
